\title{
Cost-effectiveness of the polypill versus risk assessment for prevention of cardiovascular disease
}

\author{
Bart S Ferket, ${ }_{1}^{1,2,3}$ M G Myriam Hunink, ${ }^{2,3,4}$ Mohammed Khanji, ${ }^{5}$ Isha Agarwal, ${ }^{6}$ \\ Kirsten E Fleischmann, ${ }^{7}$ Steffen E Petersen ${ }^{5}$
}

- Additional material is published online only. To view please visit the journal online (http://dx.doi.org/10.1136/ heartjnl-2016-310529)

For numbered affiliations see end of article.

Correspondence to Dr Steffen E Petersen, National Institute for Health Research Cardiovascular Biomedical Research Unit at Barts, William Harvey Research Institute, Queen Mary University of London, Charterhouse Square, London, EC1M 6BQ, UK; s.e.petersen@qmul.ac.uk

Received 27 August 2016 Revised 29 November 2016 Accepted 2 December 2016 Published Online First 11 January 2017

\section{SLinked}

- http://dx.doi.org/10.1136/ heartjnl-2016-310959

\section{CrossMark}

$$
\begin{aligned}
& \text { To cite: Ferket BS, } \\
& \text { Hunink MGM, Khanji M, } \\
& \text { et al. Heart 2017;103:491- } \\
& \text { 499. }
\end{aligned}
$$

\section{ABSTRACT}

Objective There is an international trend towards recommending medication to prevent cardiovascular disease (CVD) in individuals at increasingly lower cardiovascular risk. We assessed the cost-effectiveness of a population approach with a polypill including a statin (simvastatin $20 \mathrm{mg}$ ) and three antihypertensive agents (amlodipine $2.5 \mathrm{mg}$, losartan $25 \mathrm{mg}$ and hydrochlorothiazide $12.5 \mathrm{mg}$ ) and periodic risk assessment with different risk thresholds.

Methods We developed a microsimulation model for lifetime predictions of CVD events, diabetes, and death in 259146 asymptomatic UK Biobank participants aged 40-69 years. We assessed incremental costs and qualityadjusted life-years (QALYs) for polypill scenarios with the same combination of agents and doses but differing for starting age, and periodic risk assessment with 10-year CVD risk thresholds of 10\% and $20 \%$.

Results Restrictive risk assessment, in which statins and antihypertensives were prescribed when risk exceeded 20\%, was the optimal strategy gaining 123 QALYs (95\% credible interval (CI) -173 to 387) per 10000 individuals at an extra cost of $\mathrm{f} 1.45$ million ( $95 \% \mathrm{Cl} 0.89$ to 1.94 ) as compared with current practice. Although less restrictive risk assessment and polypill scenarios prevented more CVD events and attained larger survival gains, these benefits were offset by the additional costs and disutility of daily medication use. Lowering the risk threshold for prescription of statins to $10 \%$ was economically unattractive, costing £40 000 per QALY gained. Starting the polypill from age 60 onwards became the most cost-effective scenario when annual drug prices were reduced below $\mathrm{f240}$. All polypill scenarios would save costs at prices below $\mathrm{f} 50$. Conclusions Periodic risk assessment using lower risk thresholds is unlikely to be cost-effective. The polypill would become cost-effective if drug prices were reduced.

\section{INTRODUCTION}

Influential guideline committees recently recommended wider use of statins for the prevention of cardiovascular disease (CVD). The American College of Cardiology and American Heart Association changed their cholesterol treatment guidelines including risk assessment every 5 years using a broader CVD endpoint and a lower risk threshold for prescription. ${ }^{1}$ In the UK, the National Institute for Health and Care Excellence (NICE) updated its statin guidelines using a lower 10 -year CVD risk threshold of $10 \%$ instead of the formerly recommended $20 \%$ and extended periodic risk assessment until the age of 85 years instead of $75 .^{2}$

Instead of prescribing preventive medication based on assessment of CVD risk, a more ubiquitous approach has been proposed with a combination pill, the polypill. ${ }^{3}$ The polypill is intended for people selected only on the basis of age without the need for risk assessment. It would improve the distribution of risk factors population-wide, resulting in a tremendous decrease of CVD event rates. The polypill currently marketed consists of simvastatin $20 \mathrm{mg}$ and three half-standard dose antihypertensive agents (amlodipine $2.5 \mathrm{mg}$, losartan $25 \mathrm{mg}$ and hydrochlorothiazide $12.5 \mathrm{mg}$ ), ${ }^{4}$ and was associated with blood pressure and low-density lipoprotein cholesterol reductions in a randomised trial. ${ }^{5}$

A recent cost-effectiveness analysis demonstrated that treating low-risk individuals with statins would be optimal, but blood pressure management was not considered. ${ }^{6}$ Yet, antihypertensive treatment for individuals with lower risk or blood pressure levels has now become relevant based on the results of a large individual-level meta-analysis and the Systolic Blood Pressure Intervention Trial (SPRINT). ${ }^{7}$ At present, few cost-effectiveness analyses of the polypill approach have been performed, ${ }^{9}$ but without comparison with recommended periodic risk assessment. The aim of our study was to compare lifetime costs and health effects of the polypill approach and periodic risk assessment with different risk thresholds.

\section{METHODS}

\section{Decision model}

We developed the UK PReventiOn of Myocardial Infarction and Stroke Evaluation (UK-PROMISE) model, an individual-level state-transition (microsimulation) model. The model individualises lifetime risks for CVD, diabetes and non-CVD death based on QResearch data including 2343759 subjects. $^{10}{ }^{11}$ With the model, we simulated life courses of participants of UK Biobank, ${ }^{12}$ a large population-based study conducted in individuals recruited from the general population aged 40-69 years. UK Biobank's protocols were approved by the North West Research Ethics Committee (REC reference number: 06/ MRE08/65).

The study population was selected from 503120 UK Biobank participants attending baseline visits between 2006 and 2010. We excluded participants with stroke $(\mathrm{n}=7669)$, transient ischaemic attack (TIA) $(n=1684)$, myocardial infarction (MI) $(n=11609)$ or angina $(n=16183)$, and those using 
statin $(n=86911)$ or antihypertensive medication $(n=104032)$. In addition, participants with incomplete information on risk factors $(n=69334)$ precluding calculation of QResearch risk scores were excluded. This resulted in a study population of 259146 individuals (for baseline characteristics see table 1 and eTable 1 in the online supplementary file).

The model included health states for: 'Alive and Well', 'Alive and Diabetes Mellitus (DM)', 'Post Cardiovascular Disease (CVD) event', 'Post CVD event and DM', and 'Dead' (eFigure 1 in the online supplementary file). Each simulated individual faced a yearly probability of developing CVD, defined as: MI, angina, stroke, TIA, or dying due to causes other than CVD. The probabilities for CVD and competing non-CVD deaths were individualised using age- and gender-specific survival functions adjusted for ethnicity, social deprivation, cholesterol, body mass index, smoking, systolic blood pressure (SBP), atrial fibrillation, diabetes, chronic renal disease, rheumatoid arthritis, treated hypertension, and family history of coronary heart disease (for validity of calculated probabilities see eTable 2 in the online supplementary file). ${ }^{6}$ Case-fatality of MI and stroke was based on age and gender dependent UK statistics. After survival of CVD, modelled all-cause mortality was increased with a relative risk due to CVD. In addition, individuals without

Table 1 Baseline characteristics of UK Biobank study population

\begin{tabular}{ll}
\hline & $\begin{array}{l}\text { UK Biobank cohort } \\
\text { ( } \mathbf{n}=259146)\end{array}$ \\
\hline Age (years) & $55.0 \pm 8.1$ \\
Male gender & $111825(43.2 \%)$ \\
Smoking status & \\
Non-smoker & $127593(49.2 \%)$ \\
Former smoker & $99255(38.3 \%)$ \\
Light smoker (<10 cigarettes/day) & $15316(5.9 \%)$ \\
Moderate smoker (10-19 cigarettes/day) & $9320(3.6 \%)$ \\
Heavy smoker ( $\geq 20$ cigarettes/day) & $7662(3.0 \%)$ \\
Social deprivation (Townsend score) & $-1.4 \pm 3.0$ \\
Ethnic group & \\
White or not recorded & $246056(94.9 \%)$ \\
Indian & $2757(1.1 \%)$ \\
Pakistani & $788(0.3 \%)$ \\
Bangladeshi & $97(0.0 \%)$ \\
Other Asian & $862(0.3 \%)$ \\
Caribbean & $2162(0.8 \%)$ \\
Black African & $1575(0.6 \%)$ \\
Chinese & $804(0.3 \%)$ \\
Other & $4045(1.6 \%)$ \\
Clinical conditions & \\
Family history of early heart disease & $28102(10.8 \%)$ \\
Family history of diabetes & $23161(8.9 \%)$ \\
Type 2 diabetes & $2131(0.8 \%)$ \\
Atrial fibrillation & $912(0.4 \%)$ \\
Rheumatoid arthritis & $2276(0.9 \%)$ \\
Chronic renal disease & $112(0.0 \%)$ \\
Treated with corticosteroids & $1953(0.8 \%)$ \\
Clinical values & \\
Body mass index (kg/m ${ }^{2}$ ) & $26.8 \pm 3.9$ \\
Total cholesterol to high-density lipoprotein & $4.1 \pm 0.4$ \\
cholesterol ratio & $136.0 \pm 18.2$ \\
Systolic blood pressure (mm Hg) & \\
\hline Data are presented as mean $\pm S D$ for continuous variables and as frequencies $(\%)$ for \\
categorical data. & \\
&
\end{tabular}

diabetes had a risk of developing diabetes, based on age, gender, ethnicity, social deprivation, body mass index, smoking status, family history of diabetes, history of CVD, treated hypertension, and regular steroid use. ${ }^{11}$ The model's predictions of life expectancy until age 95 were compared with forecasts based on 2010-2012 UK life tables. ${ }^{13}$

\section{Scenarios}

We evaluated current practice, three guideline and five polypill strategies. Current practice was defined by modelled event rates assuming absence of a systematic prevention programme. We defined the guideline scenarios as: (1) 'old guidelines' recommending periodic risk assessment with prescription of statin and antihypertensive medication at 10 -year CVD risk of $20 \% ;^{14}$ (2) 'current guidelines' recommending statins at a lower $10 \%$ threshold ${ }^{2}$ and (3) 'alternative guidelines' recommending both statin and antihypertensive medication at $10 \%$. In guideline scenarios, a health check organised by the National Health Service (NHS) was offered to individuals with assessment of 10-year CVD risk every 5 years from the age of 40-75 years, unless diabetes was present or statin or antihypertensive medication was started. ${ }^{15}$ In individuals not eligible for the NHS health check, primary care physicians continued risk assessment until the age of 75 for the old guidelines and until the age of 85 for the current and alternative guidelines. An annual cardiovascular risk assessment was offered to diabetes patients free of CVD. ${ }^{16}$

Statins were offered to individuals whose 10 -year risk was above the threshold and to elderly subjects at the age of 75 for the old guidelines and age 85 for the other guidelines. Antihypertensive medication was prescribed according to hypertension guidelines when individuals with $\mathrm{SBP} \geq 140 \mathrm{~mm} \mathrm{Hg}$ had a 10-year risk above the risk threshold or when SBP was $\geq 150 \mathrm{~mm} \mathrm{Hg}$ regardless of risk. ${ }^{17}$ Uptake of health checks was based on NHS data. We modelled full uptake of cardiovascular risk assessment in diabetes patients, assuming this would be included in their annual medical examination. Adherence to preventive medication was based on cohort data. Individuals who became non-adherent to preventive medication could restart once after a new prescription within a later screening visit, but the discontinuation rate was modelled to be twice as high. After a second time of becoming non-adherent, attendance to risk assessment became zero. We assumed that statins were offered to elderly individuals only once, during a doctor's visit with the same attendance as risk assessment.

We defined five polypill scenarios in which primary care physicians would offer a polypill with the same combination of agents and doses from the age of 40, 45, 50, 55 or 60 and over. We based these starting ages on the age ranges used as inclusion criterion in combination pill trials. ${ }^{10} 18$ For each polypill scenario, we assumed that participants who initially took up risk assessment would also participate in the polypill programme. Persistence of polypill use was assumed to be the same as for statins.

\section{Treatment effects}

For antihypertensive drugs, we applied a relative CVD rate reduction associated with the expected decrease in $\mathrm{SBP}^{7}$ This decrease depended on the number and dose of the agents used. ${ }^{19}$ In guideline scenarios, the number of prescribed agents was determined by the difference between the individual's SBP at the screening visit and the target blood pressure recommended in hypertension guidelines. ${ }^{17}$ Types of prescribed antihypertensive drugs were based on the individual's age at the 
screening visit and ethnicity (for the decision making algorithm see eTable 3 in the online supplementary file). For statins we used risk ratios from a recent meta-analysis. ${ }^{20}$ If combined, we assumed absence of interaction between statin and antihypertensive drugs. We modelled the effect on developing diabetes by statin and antihypertensive drugs using odds ratios reported in meta-analyses. ${ }^{21} 22$ We assumed that subjects with serious side effects, for example, muscle pains or significant hepatic impairment, would discontinue preventive medication. In order to avoid double counting, we did not simulate occurrence of side effects and subsequent non-adherence in addition to the nonadherence rates already included in the model. More detailed information on the model is given in the eMethods and eTable 4 in the online supplementary file.

\section{Quality of life and cost data}

We included utility values dependent on age, gender and history of angina, MI or stroke as estimated for the general population. Utility values for TIA and diabetes were multiplied assuming independence. In case of CVD, a short-term disutility was subtracted. The disutility of daily preventive pill use was based on values obtained for the general population. ${ }^{23}{ }^{24}$ We estimated all costs from a UK health system perspective for the financial year 2012/2013. Polypill prices were based on Polypill.com. ${ }^{4}$
We assumed no costs and disutility when medication was discontinued. See eMethods and eTable 5 in the online supplementary file for more detailed information on utilities and costs.

\section{Analyses}

In the base-case analysis, we modelled 10000 randomly selected individuals by microsimulation and calculated events, costs, and quality-adjusted life-years (QALYs) until age 95. We discounted both costs and QALYs at an annual rate of 3.5\%. ${ }^{25}$ Incremental cost-effectiveness ratios (ICERs), defined as the difference in costs divided by the difference in QALYs, were calculated after exclusion of dominated scenarios. An ICER of less than $£ 20000-£ 30000$ per QALY gained was considered as being cost-effective. ${ }^{25}$ We calculated average net health benefits by the formula: average QALY - average cost divided by $£ 20000$ or $£ 30000$ per QALY. To calculate $95 \%$ credible intervals (CIs) and the probability of being the most cost-effective scenario, we performed a probabilistic sensitivity analysis by randomly sampling from all parameter distributions. For 500 sets of sampled parameters, the 10000 individuals were modelled accounting for population heterogeneity.

In threshold analyses we varied the costs of medication, the costs of CVD health care utilisation, the costs and relative risk of developing diabetes, and the disutility associated with

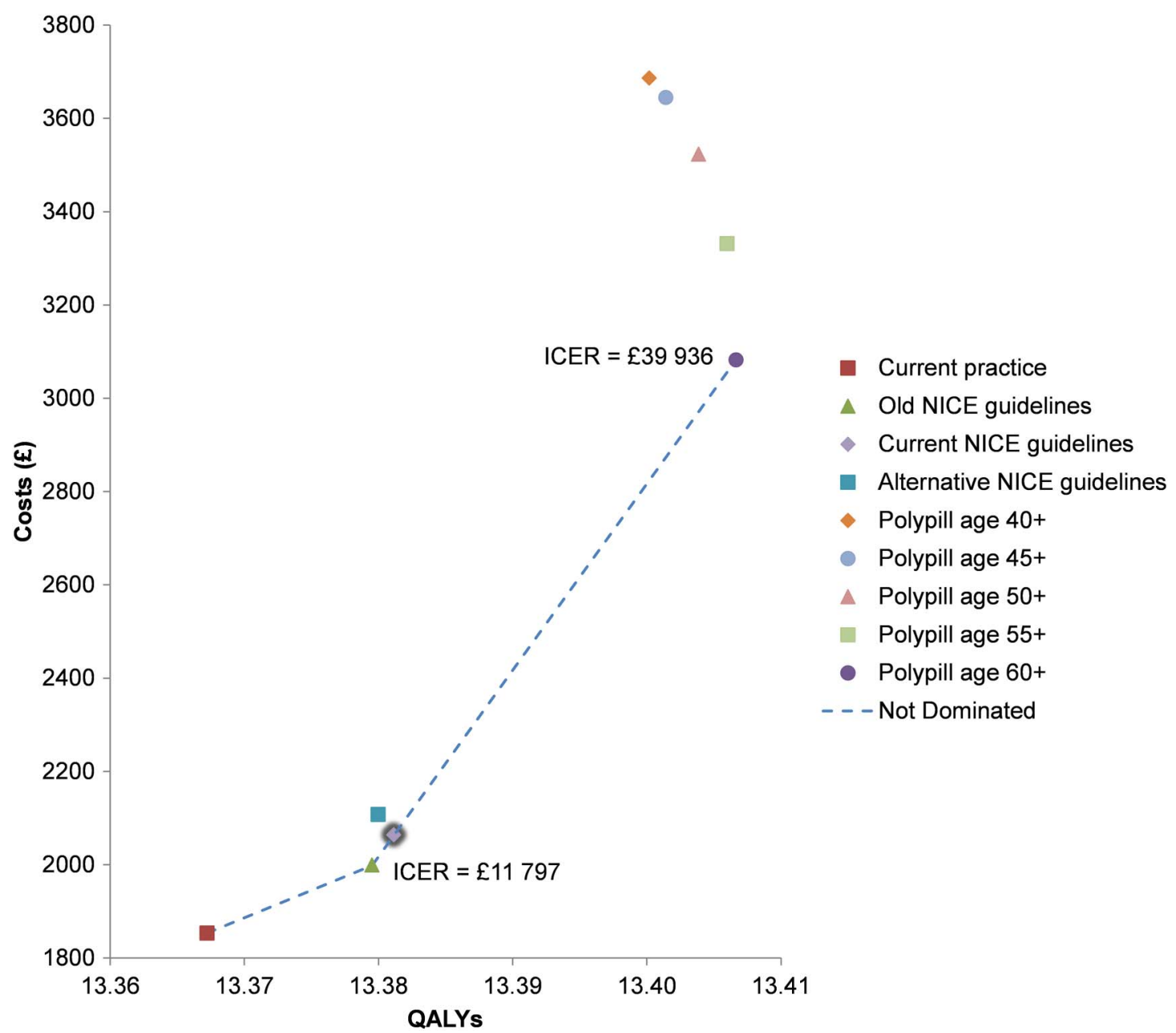

Figure 1 Cost-effectiveness graph of base-case analysis results. Costs (in UK pounds) and quality-adjusted life-years (QALYs) are means in the UK Biobank study population. The grey shaded area indicates that the scenario has been eliminated by extended dominance. Extended dominance implies that the programme is less costly than the next not absolutely dominated programme, but also has a larger incremental cost-effectiveness ratio (ICER) than this next programme. NICE, National Institute for Health and Care Excellence. 


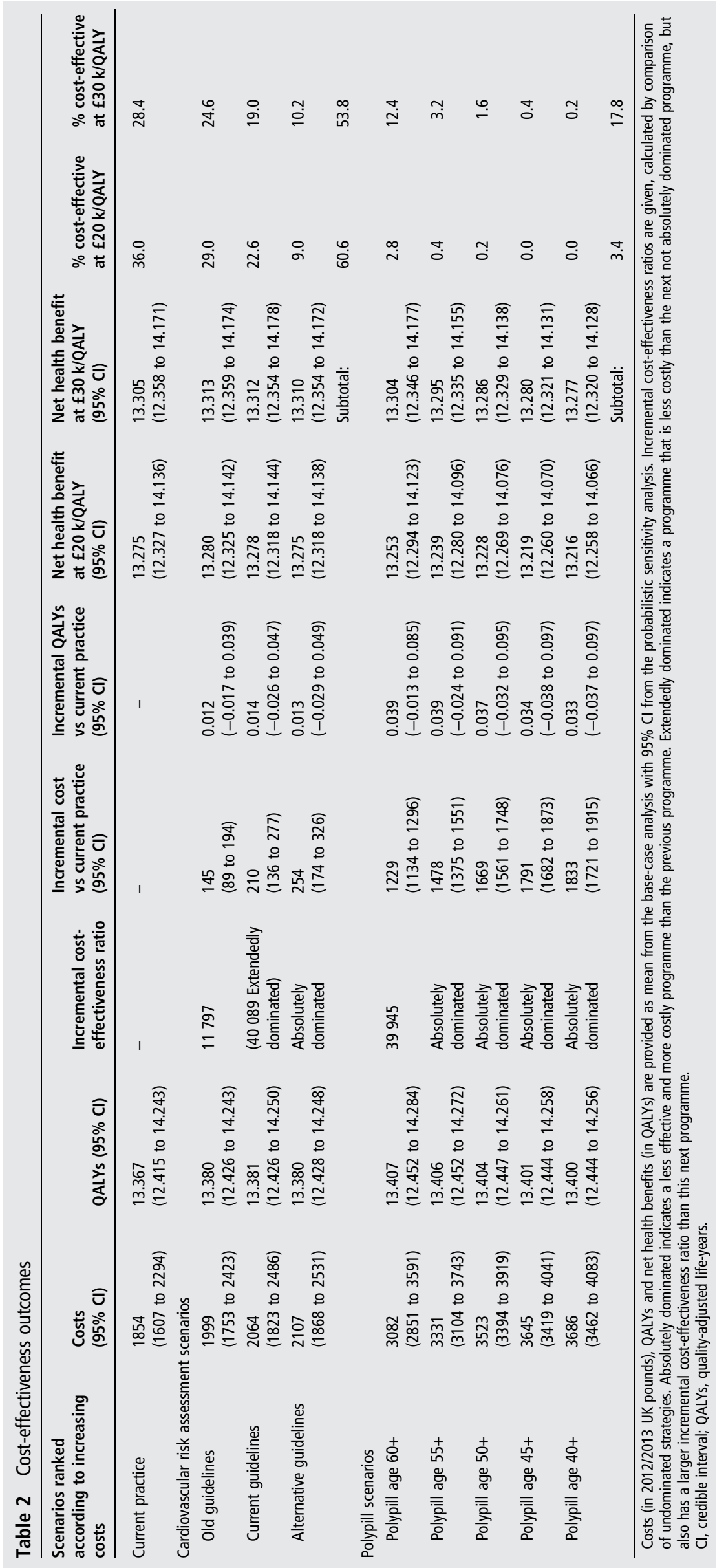


preventive pill use. Different scenarios for the uptake of prevention programmes and medication were evaluated. Furthermore, we evaluated modifications of prevention programmes by varying the stop age of periodic risk assessment and the age for starting the polypill beyond age 60 , and restricting polypill prescriptions to those with a minimal SBP of $120 \mathrm{~mm} \mathrm{Hg}$, $130 \mathrm{~mm} \mathrm{Hg}$, and $140 \mathrm{~mm} \mathrm{Hg}$ at ages of eligibility. More information on the analyses is given in the eMethods in the online supplementary file.

\section{RESULTS}

The average life expectancy estimated was 28.70 (95\% CI 28.47 to 28.99 ) vs 28.01 by life tables (also see eFigure 2 in the online supplementary file). In the base-case analysis, the old guideline scenario was the optimal strategy. This would gain 123.1 QALYs (95\% CI -173.0 to 386.8$)$ and cost $£ 1.45$ million $(0.89$ to 1.94) extra per 10000 individuals as compared with current practice with an ICER of $£ 11$ 797/QALY (figure 1 and table 2). With these old guidelines, the population's current CVD risk of $34.0 \%$ (95\% CI $33.0 \%$ to $34.9 \%$ ) would decrease by $2.6 \%$ (95\% CI $1.8 \%$ to $3.3 \%)$, whereas the diabetes incidence of $22.7 \%$ (95\% CI $21.9 \%$ to $23.5 \%$ ) would increase by $0.6 \%$ (95\% CI $-0.1 \%$ to $1.3 \%$ ) (table 3 ).

Implementation of current guidelines would result in a larger reduction of CVD risk by $0.6 \%$ (95\% CI $0.3 \%$ to $1.0 \%)$. However, as compared with the old guidelines, diabetes risk would increase by $0.2 \%$ (95\% CI $0.0 \%$ to $0.4 \%$ ) and the total number of risk assessments per 10000 individuals would increase by 3699 (95\% CI 3240 to 4222), with 1307 (95\% CI 1235 to 1368$)$ more individuals receiving a statin prescription and 502 (95\% CI 443 to 565) more receiving a prescription for antihypertensive drugs (table 3 ). The ICER was $£ 40089$ per QALY compared with old guidelines (table 2).

Polypill scenarios were more effective than risk assessment, with gains of 190 to 270 QALYs per 10000 individuals, but also more costly. Offering the polypill at age 60 was a potentially cost-effective scenario, but then a cost-effectiveness threshold of more than $£ 30000$ /QALY should be considered. Other polypill strategies were absolutely dominated, that is, less effective and more costly than if started at age 60 (figure 1 and table 2). As compared with the old guideline scenario, polypill use above age 60 reduced lifetime CVD risk further by $2.5 \%(95 \%$ CI $1.8 \%$ to $3.1 \%$ ). Also, diabetes risk was lower with polypill use: $0.2 \%$ (95\% CI $-1.7 \%$ to $1.7 \%$ ) (table 3$)$.

Superiority of the old guidelines was sensitive to the disutility of daily preventive pill use perceived as losing 6 months life expectancy. If these were to be 8 months or more, current practice was optimal, and if less than 5.5 months, current or alternative guideline scenarios were most cost-effective depending on the cost-effectiveness threshold. In addition, with a costeffectiveness threshold of $£ 30000 /$ QLLY, current guidelines were more cost-effective than old guidelines if the odds ratio of statins for increasing diabetes risk was close to 1 . These findings were furthermore sensitive to the prices of statins and antihypertensive drugs (table 4).

The polypill scenario starting at age 60 became the most costeffective strategy at annual drug prices below $£ 240$ and would become cost-saving at prices below $£ 60$ per year. All five polypill scenarios would save costs at prices below $£ 50$. Average savings would be up to $£ 1.85$ million per 10000 individuals if annual prices were $£ 10$. Starting the polypill if the individual's SBP was $\geq 140 \mathrm{~mm} \mathrm{Hg}$ or at later ages up to 75 improved its cost-effectiveness with ICERs below $£ 30000$ per QALY (table 4 and eFigure 3 in the online supplementary file).

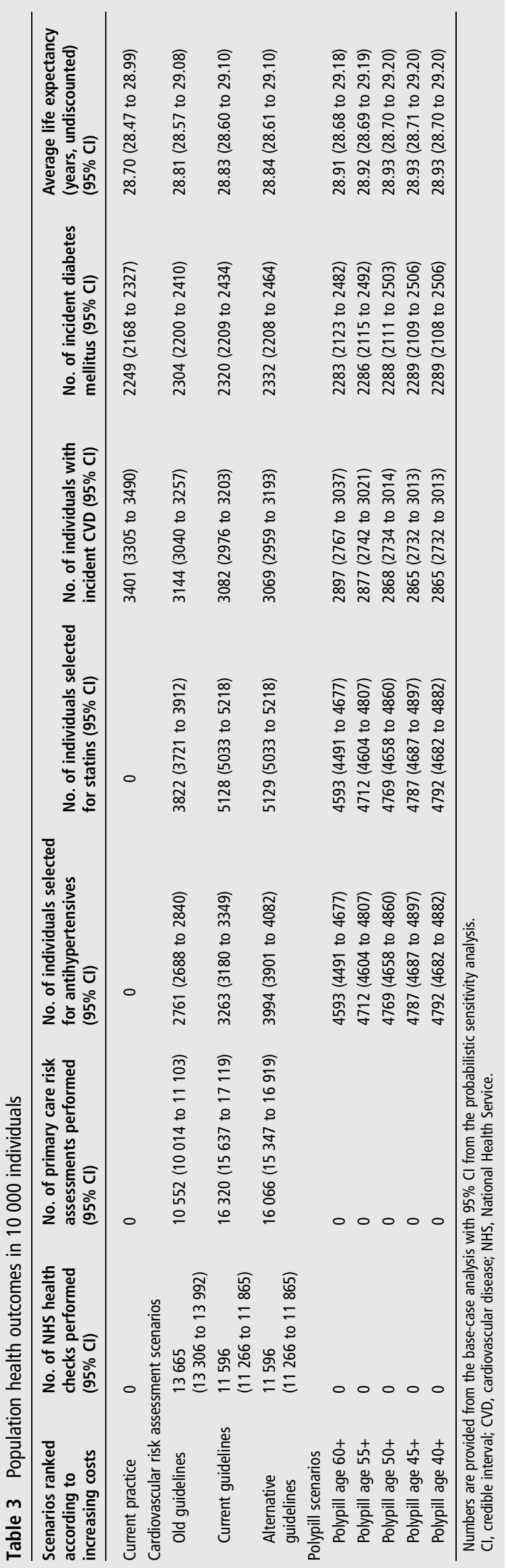


|

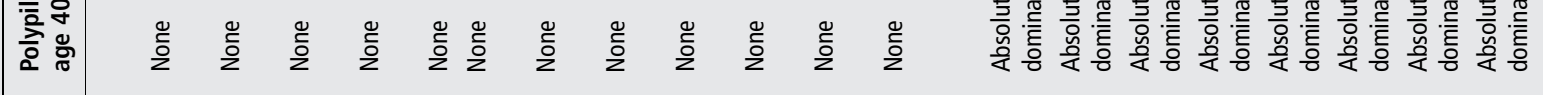

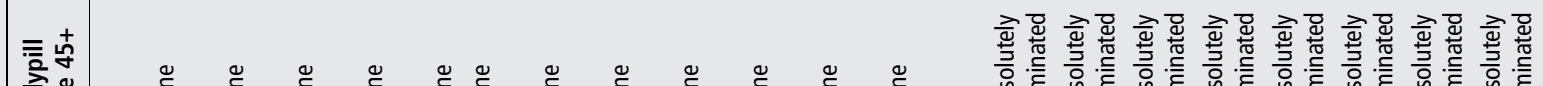

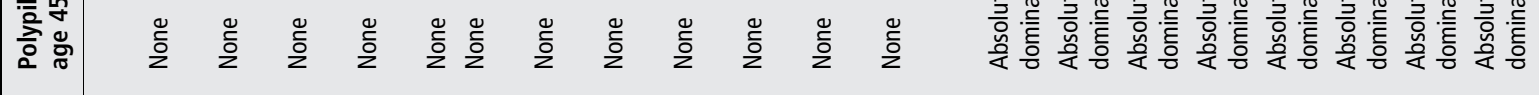

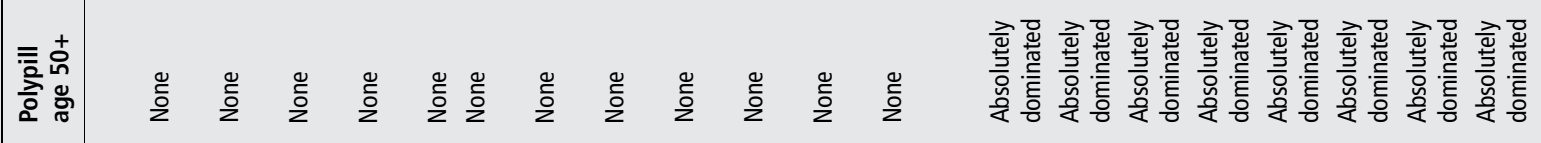

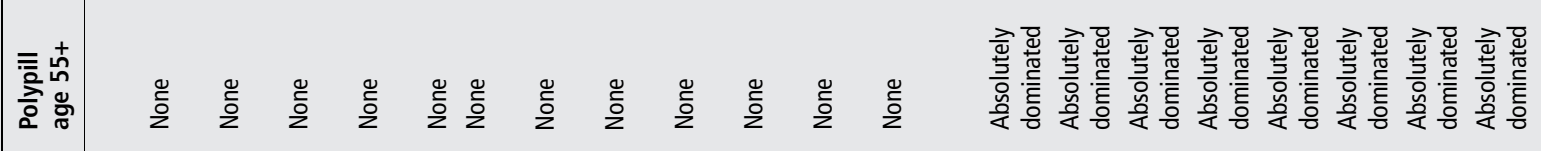

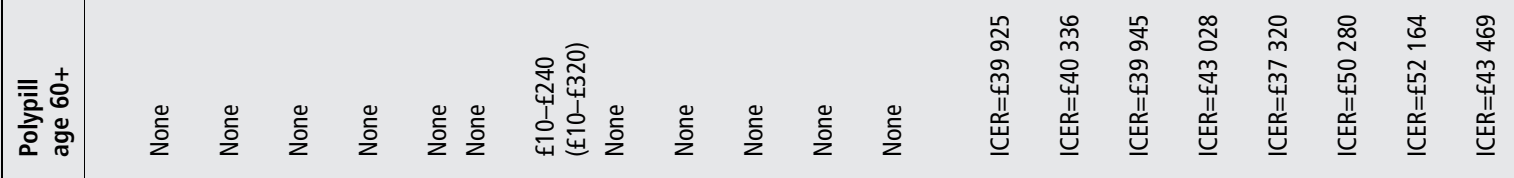

喜旁|

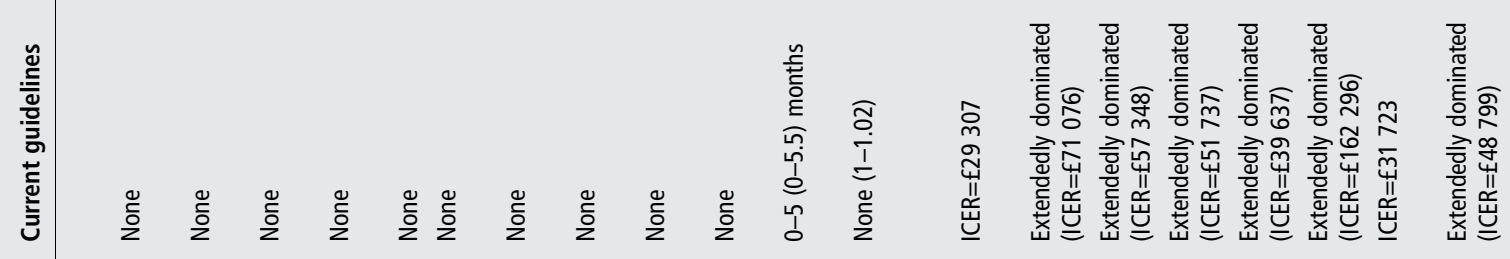

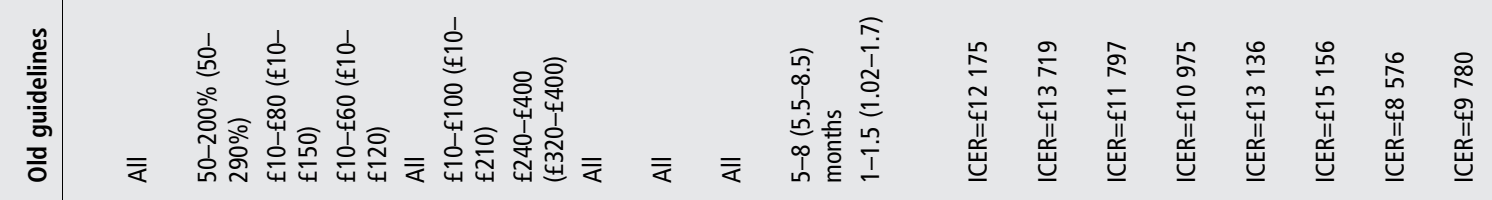

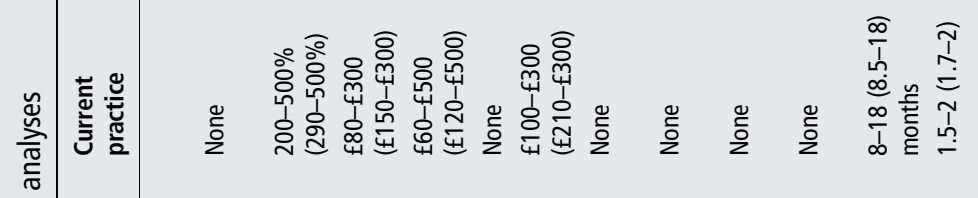

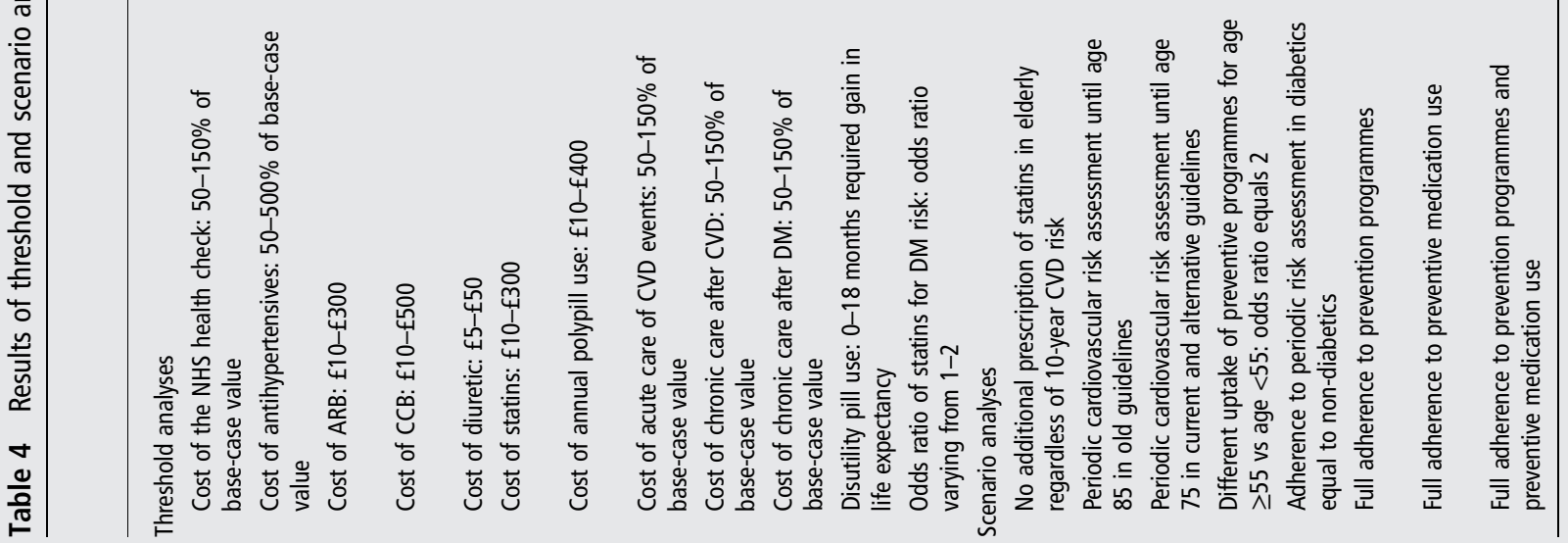




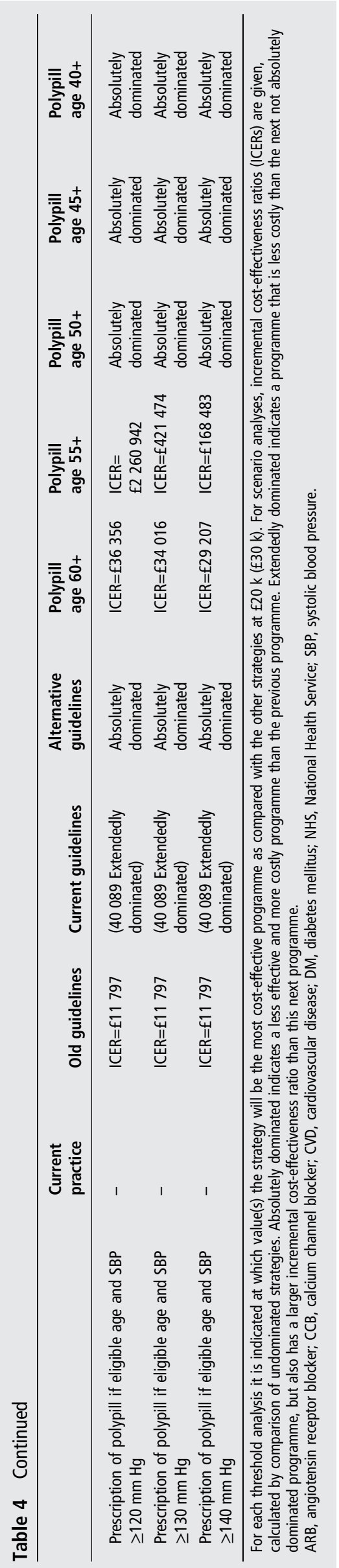

\section{DISCUSSION}

We found that periodic risk assessment with prescribing preventive drugs at a 10 -year risk of $20 \%$ was most cost-effective. Risk assessment using a lower 10-year risk threshold of $10 \%$ and polypill scenarios were more effective, in the sense that the total number of CVD events prevented and QALYs was higher. These scenarios were, however, also more costly and the incremental costs per QALY gained were high. Starting the polypill at the age of 60 and over was the most cost-effective scenario when the annual polypill price was lower than $£ 240$; polypill scenarios would save costs at prices below $£ 50$. The disutility of daily preventive medication use had a major influence on our findings, favouring the current practice scenario when this disutility was greater than or equal to losing 8 months life expectancy. Our results were furthermore sensitive to assumptions about the adverse risk of statins for development of diabetes, albeit to a lesser extent.

We demonstrated that the polypill's benefits were partly offset by the disutility of daily pill use, and its drug price outweighed potential cost savings. In contrast to our findings, two studies demonstrated that population-wide use of combination pills is cost-effective as compared with treating high-risk individuals only. ${ }^{18}{ }^{26}$ However, both studies assumed that the price of the combination pill would be the sum of the prices of its components and no pill disutility was considered.

Our analysis has a number of strengths. We predicted lifetime risks of CVD and non-CVD death using validated equations derived from 2.3 million individuals. ${ }^{6}$ To model heterogeneity in risk factor profiles, we simulated participants of a large population-based study, the UK Biobank. ${ }^{12}$ In addition, we individualised the incidence of diabetes, ${ }^{11}$ and modelled its consequences for health-related costs and quality of life. The effects of statins and antihypertensive drugs on CVD and diabetes were derived from large meta-analyses of randomised trials. ${ }^{72-22}$

Nevertheless, we had to make a number of assumptions that may have led to bias. First, we assumed that reductions in blood pressure by the polypill would follow calculations for use of the three antihypertensive drugs separately. ${ }^{19}$ Two recent meta-analyses concluded that reductions in blood pressure with the polypill are generally lower than those previously projected. ${ }^{9}{ }^{27}$ However, most of the included trials used one or two antihypertensive drugs instead of three. When the equation for estimation of the decrease in SBP with three half-dose antihypertensive drugs is applied to the average baseline SBP of the trial evaluating this polypill $(143 \mathrm{~mm} \mathrm{Hg})$, the expected decrease is similar to that observed: 18.4 vs 17.9 (95\% CI 15.7 to 20.1) $\mathrm{mm} \mathrm{Hg}$. Second, we did not explicitly model subsequent CVD events. This could contribute to an underestimation of the cost savings that can be achieved by preventing first CVD events, favouring restrictive scenarios. Nonetheless, our findings were robust within a wide range of costs associated with the care after a CVD event. Third, the increase in the odds of developing diabetes as observed in statin trials could be an artefact, because subjects who were randomised to statins had lower fatal CVD rates than subjects in the placebo group, and as a result, may have developed higher diabetes rates due to increased subject-time of follow-up. We evaluated our assumption in a sensitivity analysis, and found that if the odds ratios associated with statins were to be closer to 1 , recommendations for wider use of statins could be supported. Fourth, we did not consider differential effects of preventive medication according to the individual's characteristics and risk profile. For example, it has been reported that statins have a different effect in men and women without a history of CVD. ${ }^{28}$ Meta-analyses that also 
included the JUPITER trial, however, showed that relative reductions in coronary and stroke event rates are similar for men and women in a primary prevention setting. ${ }^{29} 30$ Nevertheless, we acknowledge that individualisation of treatment effectiveness and its implications for the design and content of prevention programmes should be further investigated.

There is an international trend towards recommending medication use to prevent CVD in individuals at lower cardiovascular risk. ${ }^{12}$ It has been estimated that the new US cholesterol treatment guidelines will increase the number of adults eligible for statins by $11 \%{ }^{31}$ Around the world these changes to guidelines have sparked the debate on 'over-medicalization'. 32 We now provide evidence that expanding the use of statins and antihypertensive drugs for CVD prevention appears to improve survival but that the incremental costs per QALY gained are high, mainly because the general population assigns a small quality of life decrement to preventive pill use. Therefore, future research should compare the cost-effectiveness of non-pharmacological preventive strategies to interventions that give rise to long-term medication use.

\section{CONCLUSION}

Periodic risk assessment with lower risk thresholds to initiate preventive drugs is unlikely to be cost-effective. A population approach with the polypill would become cost-effective if drug prices were reduced.

Key messages

\section{What is already known on this subject?}

- Guideline committees recently changed their recommendations for periodic cardiovascular risk assessment, which will potentially result in wider use of drugs for the primary prevention of cardiovascular disease.

- As an alternative to periodic risk assessment, a population approach with a polypill including a statin and three half-standard dose antihypertensive agents has been proposed; this polypill was associated with significant cardiovascular risk factor reductions in a randomised trial.

\section{What might this study add?}

- Periodic cardiovascular risk assessment using a restrictive threshold for prescription of statins and antihypertensive drugs, as recommended in the guidelines before the updates, is most cost-effective.

\section{How might this impact on clinical practice?}

- A population approach with the polypill is currently too costly to be implemented in the UK, but would become cost-effective if drug prices were reduced.

\section{Author affiliations}

${ }^{1}$ Department of Population Health Science and Policy, Institute for Healthcare Delivery Science, Icahn School of Medicine at Mount Sinai, New York, New York, USA

${ }^{2}$ Department of Epidemiology, Erasmus MC, Rotterdam, The Netherlands

${ }^{3}$ Department of Radiology, Erasmus MC, Rotterdam, The Netherlands

${ }^{4}$ Center for Health Decision Science, Harvard T.H. Chan School of Public Health, Boston, Massachusetts, USA

${ }^{5}$ William Harvey Research Institute and NIHR Cardiovascular Biomedical Research Unit at Barts, Queen Mary University of London, London, UK

${ }^{6}$ Department of Epidemiology, Harvard T.H. Chan School of Public Health, Boston,
Massachusetts, USA

Division of Cardiology, UCSF Medical Center, San Francisco, California, USA

Acknowledgements This research has been conducted using the UK Biobank Resource.

Contributors BSF, MGMH, MK, IA, KEF, SEP all had substantial contributions to the conception and design of the work, the acquisition, analysis, and interpretation of data for the work, the drafting of the work or revising it critically for important intellectual content. BSF, SEP, and MK searched the literature; BSF and SEP developed the decision model and performed the simulations; BSF, MGMH, MK, and SEP analysed the data. SP is the guarantor.

Funding This work was directly funded by the National Institute for Health Research Cardiovascular Biomedical Research Unit at Barts.

Competing interests None declared.

Ethics approval UK Biobank's protocols were reviewed and approved by the North West Research Ethics Committee (REC Reference Number: 06/MRE08/65).

Provenance and peer review Not commissioned; externally peer reviewed.

Data sharing statement Statistical codes and patient level data are available to researchers who apply to use the UK Biobank Resource at http://www.ukbiobank.ac uk/register-apply/ through UK Biobank's Access Procedures, which has a protocol for approving data requests. Because of restrictions based on privacy regulations and informed consent of the participants, data cannot be made freely available in a public repository.

\section{REFERENCES}

1 Stone NJ, Robinson JG, Lichtenstein AH, et al. 2013 ACC/AHA guideline on the treatment of blood cholesterol to reduce atherosclerotic cardiovascular risk in adults: a report of the American College of Cardiology/American Heart Association Task Force on Practice Guidelines. J Am Coll Cardiol 2014;63(25 Pt B):2889-934.

2 National Institute for Health and Care Excellence. Lipid modification: cardiovascular risk assessment and the modification of blood lipids for the primary and secondary prevention of cardiovascular disease. Issued July 2014. London: National Institute for Health and Care Excellence, 2014.

3 Wald NJ, Law MR. A strategy to reduce cardiovascular disease by more than $80 \%$. BMJ 2003:326:1419.

4 Polypill.com. https://www.polypill.com/ (accessed 15 Jun 2016).

5 Wald DS, Morris JK, Wald NJ. Randomized polypill crossover trial in people aged 50 and over. PLoS One 2012;7:e41297.

6 Pandya A, Sy S, Cho S, et al. Cost-effectiveness of 10-year risk thresholds for initiation of statin therapy for primary prevention of cardiovascular disease. JAMA 2015:314:142-50.

7 Sundström J, Arima H, Woodward M, et al., Blood Pressure Lowering Treatment Trialists' Collaboration. Blood pressure-lowering treatment based on cardiovascular risk: a meta-analysis of individual patient data. Lancet 2014:384:591-8.

8 Wright JT Jr, Williamson JD, Whelton PK, et al., SPRINT Research Group. A randomized trial of intensive versus standard blood-pressure control. N Engl J Med 2015;373:2103-16.

9 Elley CR, Gupta AK, Webster R, et al. The efficacy and tolerability of 'polypills': meta-analysis of randomised controlled trials. PLoS One 2012;7:e52145.

10 Hippisley-Cox J, Coupland C, Robson J, et al. Derivation, validation, and evaluation of a new QRISK model to estimate lifetime risk of cardiovascular disease: cohort study using QResearch database. BMJ 2010;341:c6624.

11 Hippisley-Cox J, Coupland C, Robson J, et al. Predicting risk of type 2 diabetes in England and Wales: prospective derivation and validation of QDScore. BMJ 2009:338:b880.

12 Sudlow C, Gallacher J, Allen N, et al. UK Biobank: an open access resource for identifying the causes of a wide range of complex diseases of middle and old age. PLoS Med 2015;12:e1001779.

13 Office for National Statistics. National Life Tables, 2010-2012. http://www.ons.gov. uk/ons/rel/lifetables/national-life-tables/2010---2012/index.html (accessed 15 Jun 2016)

14 National Institute for Health and Care Excellence. Lipid modification: cardiovascular risk assessment and the modification of blood lipids for the primary and secondary prevention of cardiovascular disease. Updated in 2010. London: National Institute for Health and Care Excellence, 2008.

15 NHS Health Check. http://www.healthcheck.nhs.uk/ (accessed 15 Jun 2016).

16 National Institute for Health and Care Excellence. Type 2 diabetes. The management of type 2 diabetes. Updated in 2014. London: National Institute for Health and Care Excellence, 2009.

17 National Institute for Health and Clinical Excellence. Hypertension: the clinical management of primary prevention in adults. London: National Institute for Health and Clinical Excellence, 2011 
18 Rubinstein A, Garcia Martí S, Souto A, et al. Generalized cost-effectiveness analysis of a package of interventions to reduce cardiovascular disease in Buenos Aires, Argentina. Cost Eff Resour Alloc 2009;7:10.

19 Law MR, Morris JK, Wald NJ. Use of blood pressure lowering drugs in the prevention of cardiovascular disease: meta-analysis of 147 randomised trials in the context of expectations from prospective epidemiological studies. BMJ 2009;338: b1665.

20 Taylor $F$, Huffman MD, Macedo AF, et al. Statins for the primary prevention of cardiovascular disease. Cochrane Database Syst Rev 2013;(1):CD004816.

21 Sattar N, Preiss D, Murray HM, et al. Statins and risk of incident diabetes: a collaborative meta-analysis of randomised statin trials. Lancet 2010;375:735-42.

22 Elliott WJ, Meyer PM. Incident diabetes in clinical trials of antihypertensive drugs: a network meta-analysis. Lancet 2007;369:201-7.

23 Fontana $\mathrm{M}$, Asaria $\mathrm{P}$, Moraldo $\mathrm{M}$, et al. Patient-accessible tool for shared decision making in cardiovascular primary prevention: balancing longevity benefits against medication disutility. Circulation 2014;129:2539-46.

24 Hutchins R, Viera AJ, Sheridan SL, et al. Quantifying the utility of taking pills for cardiovascular prevention. Circ Cardiovasc Qual Outcomes 2015;8:155-63.

25 National Institute for Health and Care Excellence. Guide to the methods of technology appraisal 2013. Process and methods guides. National Institute for Health and Care Excellence, 2013.
26 van Gils PF, Over EA, Hamberg-van Reenen $\mathrm{HH}$, et al. The polypill in the primary prevention of cardiovascular disease: cost-effectiveness in the Dutch population. BMJ Open 2011;1:e000363.

27 de Cates AN, Farr MR, Wright N, et al. Fixed-dose combination therapy for the prevention of cardiovascular disease. Cochrane Database Syst Rev 2014;(4) CD009868.

28 Petretta $\mathrm{M}$, Costanzo P, Perrone-Filardi $\mathrm{P}$, et al. Impact of gender in primary prevention of coronary heart disease with statin therapy: a meta-analysis. Int J Cardiol 2010;138:25-31.

29 Brugts JJ, Yetgin T, Hoeks SE, et al. The benefits of statins in people without established cardiovascular disease but with cardiovascular risk factors: meta-analysis of randomised controlled trials. BMJ 2009;338:b2376.

30 Kostis WJ, Cheng JQ, Dobrzynski JM, et al. Meta-analysis of statin effects in women versus men. J Am Coll Cardiol 2012;59:572-82.

31 Pencina MJ, Navar-Boggan AM, D'Agostino RB Sr, et al. Application of new cholesterol guidelines to a population-based sample. $N$ Engl J Med 2014;370:1422-31.

32 Ioannidis JP. More than a billion people taking statins?: Potential implications of the new cardiovascular guidelines. JAMA 2014;311:463-4.

33 Wise J. Open letter raises concerns about NICE guidance on statins. BMJ 2014;348: g3937.

\section{IMAGE CHALLENGE}

\section{A case of recent myocardial infarction with cardiac failure}

\section{CLINICAL INTRODUCTION}

A 50-year-old hypertensive smoker presented with a typical angina of 2 days duration. An urgent ECG revealed extensive anterior wall myocardial infarction. In view of the delayed presentation, the patient was conservatively managed with heparin. In-hospital echocardiogram showed akinesia of entire left anterior descending artery (LAD) territory with severe left ventricular (LV) dysfunction. He was discharged with a plan for early coronary intervention. However, he presented a fortnight later with acute pulmonary oedema. General appraisal revealed a restless individual who was dyspnoeic and diaphoretic at rest. On clinical examination, the patient was in hypotension with features of biventricular failure. A 12-lead ECG showed QS pattern with persistent ST segment elevation in precordial leads. The chest radiograph demonstrated features of pulmonary oedema, cardiomegaly and bilateral pleural effusion. Creatine PhosphokinaseMB (CPK-MB) was negative. A preliminary transthoracic echocardiography was done (figure 1 and see online supplementary video 1 ).

\section{QUESTION}

What is the most likely diagnosis based on the echocardiogram?

A. LV pseudo-aneurysm with contained rupture

B. Dissecting intramural haematoma of LV apex

C. Ventricular apical aneurysm with thrombus

D. LV non-compaction with prominent ventricular trabaculations For the answer see page 529
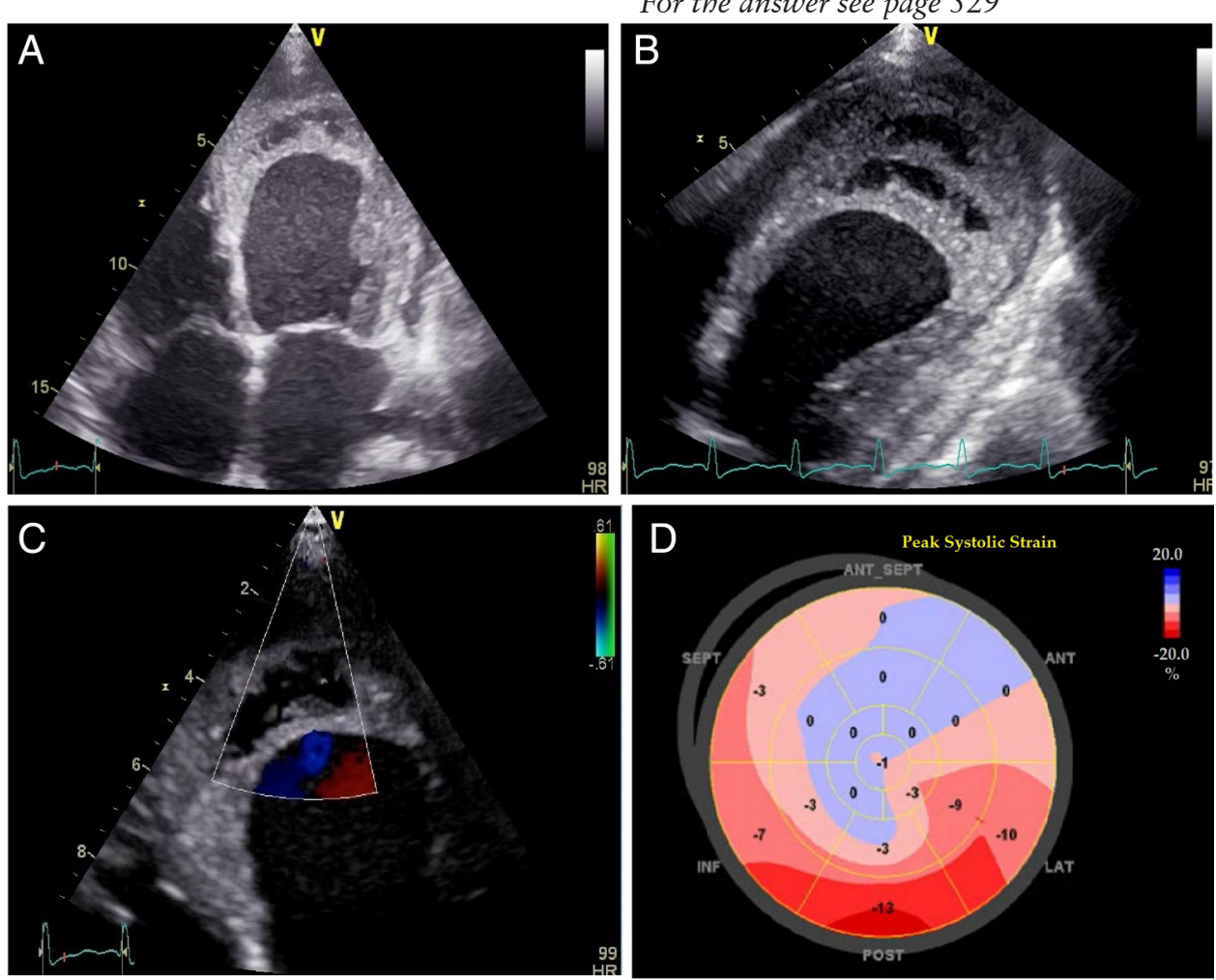

Figure 1 (A) Trans thoracic echocardiogram-apical 4 chamber view; (B) zoomed view of apex; (C) colour Doppler at left ventricular (LV) apex and (D) bulls-eye plot of strain imaging. 